\title{
Exploring light propagating in photonic crystals with Fourier optics
}

\author{
Nicolas Le Thomas, ${ }^{1}{ }^{*}$ Romuald Houdré, ${ }^{1}$ Maria V. Kotlyar, ${ }^{2}$ David O’Brien, ${ }^{2}$ and Thomas F. Krauss ${ }^{2}$ \\ ${ }^{1}$ Institut de Photonique et d'Électronique Quantiques, École Polytechnique Fédérale de Lausanne (EPFL), \\ CH-1015 Lausanne, Switzerland \\ ${ }^{2}$ SUPA, School of Physics and Astronomy, University of St. Andrews, St. Andrews, Fife KY16 9SS, UK \\ *Corresponding author: nicolas.lethomas@epfl.ch
}

Received August 3, 2007; accepted September 26, 2007;

posted October 10, 2007 (Doc. ID 86072); published November 14, 2007

Photonic crystals (PhCs) act on light in two different ways: confinement and modification of propagation. Both phenomena rely on the complex interplay between multiply scattered waves that can form what is known as a Bloch mode. Here, we present a technique that allows direct imaging of Bloch modes, both in real space and in $k$-space. The technique gives access to the location of the field maxima inside the $\mathrm{PhC}$, the dispersion relation, equifrequency surfaces, as well as reflection and transmission coefficients. Our key advance is that we retrieve the desired information comprehensively, without postprocessing or cumbersome near-field scanning techniques, even for modes that are nominally lossless, i.e., below the light line. To highlight the potential of the technique, we extract the dispersion curve of a coupled cavity waveguide consisting of as many as 100 cavities, as well as the equifrequency surfaces and polarization properties of a PhC beam splitter. (C) 2007 Optical Society of America

OCIS codes: $070.0070,130.0130,130.2790,130.5990$.

\section{INTRODUCTION}

Planar photonic crystals (PhCs) [1] are defined by a periodic modulation of the refractive index, with a periodicity of order 300-500 $\mathrm{nm}$ for applications in optical communications. The standard characterization technique is to assess their transmission as a function of wavelength [1]. While this technique yields a lot of useful information and can be combined with interferometric techniques to determine the group velocity of the propagating wave [2,3], it does not reveal the complete nature of the propagation inside the structure, i.e., it is a type of black box approach. Furthermore, the two most important tools for describing $\mathrm{PhCs}$, namely the band structure and the equifrequency surfaces (EFSs), need to be inferred from numerical calculation. The band structure, i.e., the $\omega-k$ relationship of the allowed states of the system, indicates the presence of bandgaps, the group velocity of Bloch modes, and their polarization dependence. The EFSs are derived from the band structure and describe the direction of propagation for a given mode at a fixed frequency [4,5]. To fully understand photonic PhCs and their Bloch modes, it is then desirable to extract the band structure and EFS from direct observation and measurement. This has motivated several research groups [6-10] to develop scanning near-field optical microscopy (SNOM) for this purpose, coupled with numerical analysis to extract the band structure. The SNOM can see Bloch modes by probing their evanescent fields, and achieve high spatial resolution, but the inherent need to perform point-by-point image scanning makes the method cumbersome and time consuming. Imaging the Bloch modes directly is much faster and simpler, but is a technique that has been mainly neglected so far, with a few exceptions [11-14]. By accessing the focal plane of a low numerical aperture microscope objective, Fourier imaging has also been used to reveal the band structure of a square photonic lattice directly [14]. To take this work further and provide comprehensive evidence for the power of the direct imaging technique we show how, in combination with Fourier space imaging based on a high numerical aperture objective, direct imaging can be used to fully characterize the properties of Bloch modes. The characterization includes field distribution, transmission/ reflection coefficients at interfaces, band structures, and EFSs. We thereby prove that conventional optical methods can reveal a host of information with remarkable speed and accuracy. Our technique will open Bloch mode imaging to a much wider audience and thereby accelerate the development of $\mathrm{PhCs}$ and periodic structures in general.

Many original concepts in periodic structures [15] can be directly observed above the light line, i.e., without the need for evanescent mode detection, including negative refraction [16,17], the superprism [18], and focusing effects [19] as well as self-collimation [20]. In all cases, observation of the propagation direction, the dispersion curves, and the EFSs are of primary importance as well as the finite size of both the $\mathrm{PhC}$ and the exciting beam, as pointed out in $[4,5]$. For minimal losses application, operation below the light line is more appealing. Even with devices designed to operate below the light line, truly lossless propagation is in practice impossible, as most photonic devices do exhibit finite-size effects, for instance. From a general point of view, a careful physical investigation of the losses permits retrieving fundamental information about the light propagation in all kinds of photonic devices. To underline this point and to expose the full 
potential of Fourier space imaging, we demonstrate its capabilities on four different types of nanophotonic structures: a self-collimating square lattice tile, a polarization beam splitter operating above the light line, a W1 waveguide, and a coupled cavity waveguide made of 100 cavities operating below the light line.

\section{EXPERIMENTAL SETUP}

We use a standard end-fire setup to excite the $\mathrm{PhC}$ structures and to obtain their transmission spectra. The setup consists of a tunable laser diode operating at $1.55 \mu \mathrm{m}$ with a wavelength resolution $\Delta \lambda=0.001 \mathrm{~nm}$, a polarization controller, microlensed fibers, and an InGaAs power meter. The light emitted from the $\mathrm{PhC}$ top surface is collected by a high numerical aperture $(\mathrm{NA}=0.9)$ objective and is imaged in an intermediate imaging plane (Fig. 1) prior to detection with an InGaAs camera either in the near field or in the far field with an angular range of $\pm 64^{\circ}$ (blue and green paths in Fig. 1, respectively). Note that from now on, we will use the terms near-field imaging and far-field imaging to refer to imaging in real space and in Fourier space, respectively. The objective $\mathrm{O}$ performs the Fourier transform of the object plane into the Fourier plane $\xi$, where an aperture sets the limits in the bandwidth of the transmitted spatial frequencies. The lens $\mathrm{L}_{1}$ performs another Fourier transform and creates an image in the intermediate image plane $x$, allowing spatial filtering. Switching between the near-field and the far-field images is accomplished by removing only the optical lens $\mathrm{L}_{2}$ : either the intermediate image plane $x$ or the Fourier plane $\xi$ is imaged on the CCD array with and without the lens $\mathrm{L}_{2}$, respectively.

\section{REAL-SPACE AND FOURIER SPACE IMAGING OF PhC OPERATING IN THE SELF-COLLIMATION REGIME}

One of the simplest types of planar $\mathrm{PhCs}$, which will serve as a model system, is a square lattice of holes etched in a two-dimensional (2D) waveguide. Some of the unique properties of Bloch waves are already apparent in these simple tiles. For instance, light can propagate without detectable diffraction over centimeter-scale distances, as recently demonstrated [21]. This is due to the supercollimation effect that is characterized by a square-shaped EFS (Fig. 2). As the direction of a propagating Bloch mode is

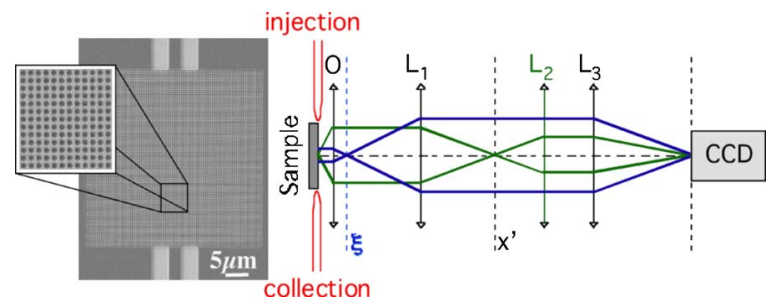

Fig. 1. (Color online) Experimental setup; O microscope objective (NA 0.9), $\mathrm{L}_{1}, \mathrm{~L}_{2}$, and $\mathrm{L}_{3}$ achromatic lenses and on the left electron microscopy image of a $\mathrm{PhC}$ tile with the access ridge waveguides. The green path corresponds to the near-field imaging and the blue corresponds to the far-field imaging with $\mathrm{L}_{2}$ removed. $\xi$ and $x$ are the Fourier plane and intermediate image plane, respectively. always normal to the local EFS [21], self-collimation is achieved when the EFSs are locally as flat as possible. In Figs. 2(a)-2(d) the infrared light propagating through an 80 period square lattice tile is imaged in the near field and the far field with high spatial and frequency resolution. The lattice (lattice parameter $a=0.45 \mu \mathrm{m}$, hole radius $r=0.127 \mu \mathrm{m})$ is designed to operate in the selfcollimation regime for TM polarized light with $\lambda$ $\sim 1.55 \mu \mathrm{m}$. The GaInAsP/InP planar waveguide, made of a $522 \mathrm{~nm} \mathrm{Ga} \mathrm{Ga}_{1-x} \mathrm{As}_{y} \mathrm{P}_{1-y}$ core layer sandwiched between two InP cladding layers of thickness 300 (upper) and $600 \mathrm{~nm}$ (lower), respectively [22], and the access ridge waveguides of width $d=3 \mu \mathrm{m}$ (see the inset of Fig. 1) are single mode in the frequency range of interest. For a wavelength of $1475 \mathrm{~nm}$ (normalized frequency parameter $u=a / \lambda \sim 0.305$ ), the mode at the access waveguide output is diffracted in the $2 \mathrm{D}$ plane with an angle $\theta_{\text {diff }} \sim \lambda /(n$ $\times d) \sim 10$ degrees, with $n$ the effective index of the planar waveguide. In this regime, the $\mathrm{PhC}$ acts as an isotropic medium. When the wavelength increases, we enter the self-collimation regime and the beam propagates without diffraction at $\lambda=1550 \mathrm{~nm} ; u=0.29$. In addition to the selfcollimation effect, the near-field intensity pattern measured in Figs. 2(a)-2(d) exhibits an intensity modulation along the $X$ direction whose associated spatial frequency increases for decreasing normalized frequency $u$. Based on Fourier space imaging, we can prove that this modulation arises from the standing wave produced by constructive interference between the forward (FW) and the backward (BW) propagating Bloch waves in the structure.

The in-plane component $\vec{k}_{/ /}=\vec{k}_{x}+\vec{k}_{y}$ of the propagating Bloch mode is directly recorded in the Fourier space images shown in Figs. 2(e)-2(h) (for more details refer to the Appendix). We observe a main spot located at $-k_{x}$ and elongated along the $k_{y}$ direction. It is attributed to a forward propagating Bloch wave [23]. For short wavelengths/high frequencies, the measured spot pattern is crescent shaped, whereas for frequencies in line with the self-collimation regime, it becomes a straight vertical line as expected from the theoretical EFS [20,21]. The length of the curve in the $k_{y}$ direction is inversely proportional to the spatial extent of the Bloch mode, which is directly related to the access waveguide width. Assuming a fill factor of $f=25 \%$ for the experimental structure, the experimentally deduced dispersion curves $\omega(k)$ at $k_{y}=0$ for the TE and TM polarized modes are in good agreement with a $2 \mathrm{D}$ plane wave expansion (PWE) calculation for a TE and TM effective index of 3.28 and 3.22, respectively, as shown in Fig. 3.

The spot symmetrically located at $+k_{x}$ in Figs. $2(\mathrm{e})-2(\mathrm{~h})$ reveals the presence of a BW propagating Bloch wave, which results mainly from the reflection at the air-ridge waveguide interface. The intensity ratio $R \sim 0.15$ at $\lambda$ $=1.55 \mu \mathrm{m}$ between the $-k_{x}$ and $+k_{x}$ waves is in line with the fringe visibility of the spatial modulation observed in real space. Consequently, this modulation, whose periodicity is directly related to $k_{x}$, is simply the standing wave or Fabry-Perot-type effect. The real-space fringe spacing $\Delta x$ of $5 \times a=2.25 \mu \mathrm{m}$ observed in Fig. 2(b) agrees with the spatial beating frequency $\Delta k$ of $0.2 \times 2 \pi / a=2 \pi / \Delta x$ $=2.8 \mu \mathrm{m}^{-1}$ observed in Fig. 2(f). We would like to stress that without any reflection, a constant intensity pattern 

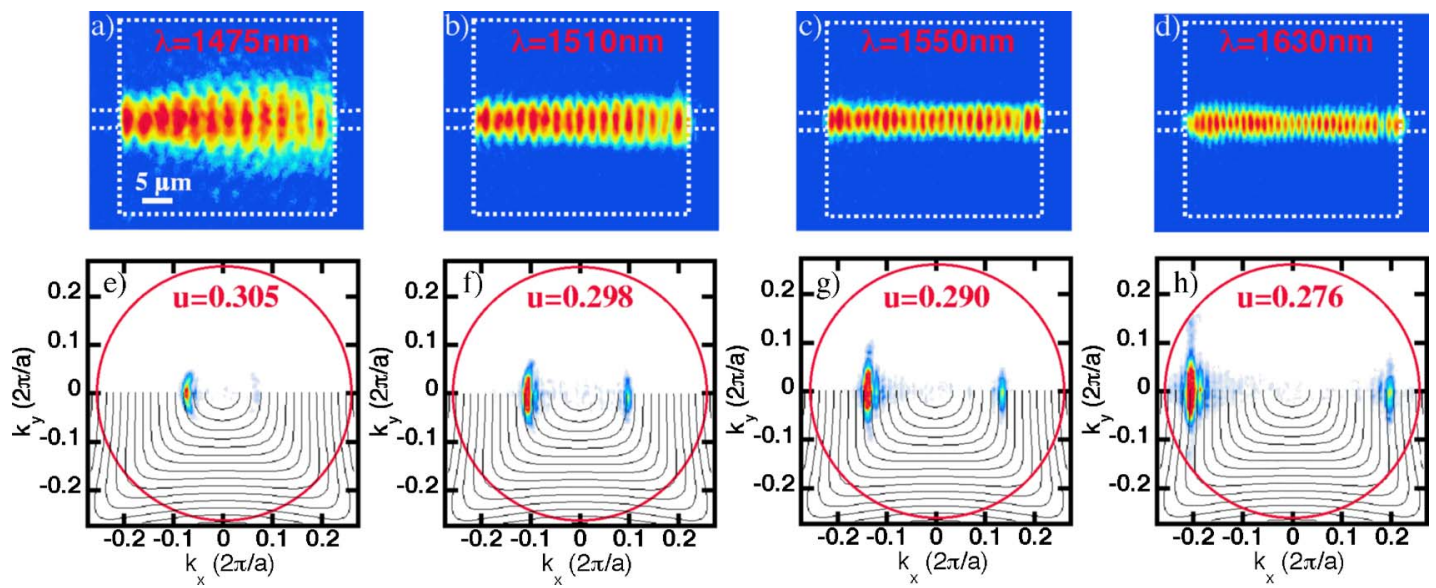

Fig. 2. (Color online) (a)-(d) Near-field image of the Bloch wave mode in a square lattice PhC tile of 80 periods for different reduced energies $u$. Below each real space image is shown the corresponding far-field image from (e) to (h). The thin black curves in the lower half space of the far-field frame are the 2D PWE theoretical EFSs starting from $u=0.315$ in the center and drawn with a step of -0.005 . The far-field image is limited by the maximum propagating wave vector $k_{\max }=0.2636 \times 2 \pi / a$ (the red circle in Figs. $2(\mathrm{e})-2(\mathrm{~h})$ delimits the output pupil of the objective that defines $k_{\max }$ ). The corresponding diffraction limited spatial resolution is $0.85 \mu \mathrm{m}$.

would be measured. As a result, without these interferences, the phase information and the dispersion relation curve would be lost in real space. In Fourier space, however, the spot at position $-k_{x}$ would still be present. Fourier space imaging is therefore a more universal and straightforward method to retrieve the dispersion relation than real-space imaging. Moreover, FW and BW propagating waves cannot be separated in real-space imaging as only the intensity of the field is recorded, while the counterpropagating wave can be filtered in $k$-space allowing, for instance, recovery of the loss profile of the forward propagating wave.

\section{PROBING DISPERSION PROPERTIES BELOW LIGHT CONE FROM SIZE EFFECTS}

The far-field characterization of infinite $\mathrm{PhC}$ structures cannot record information carried by Bloch waves whose

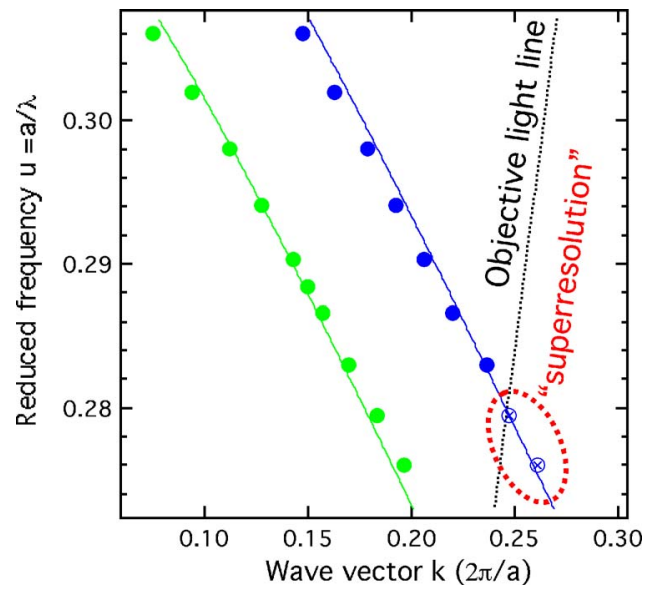

Fig. 3. (Color online) Dispersion curves for a square lattice $\mathrm{PhC}$ for TE (blue) and TM (green) polarization, plotted versus the modulus of the wave vector. The points represent the experimental data whereas the lines are for the theoretical PWE calculation $\left(f=25 \%, n_{\text {eff }}^{T E}=3.28\right.$, and $\left.n_{\text {eff }}^{T M}=3.22\right)$. The dotted line is the light line of the microscope objective. Two experimental data points below this light line are highlighted with circle-cross points. The horizontal error bars are given by the size of the points while the vertical ones are far below the size of the points. wave vector lies below the light cone, except if a noninvasive local probe or properly designed perturbations convert the evanescent field into radiative fields [24]. Finite size or light localization implies, however, that the Fourier space spectrum broadens and that some spatial frequencies of such Bloch waves fall within the light cone, as shown in Figs. 4 and 5, respectively. In Fig. 4, the broadening of the far-field spectrum is highlighted with three square lattice $\mathrm{PhC}$ tiles (with structural parameters identical to the tile studied in Section 3) operating in the selfcollimating regime $(u=0.283)$ that differ by their size of 9 , 18 , and $36 \mu \mathrm{m}$, respectively. The emitted light is diffracted due to the finite size of the tile along the light propagation direction defined by the $\mathrm{PhC}$ line defect. As a result, the peak signal in Fourier space, which is a $\delta$ function along $k_{x}$ for an infinite structure, broadens along this direction into a sinc function that has secondary maxima that are located into the light cone. Such secondary

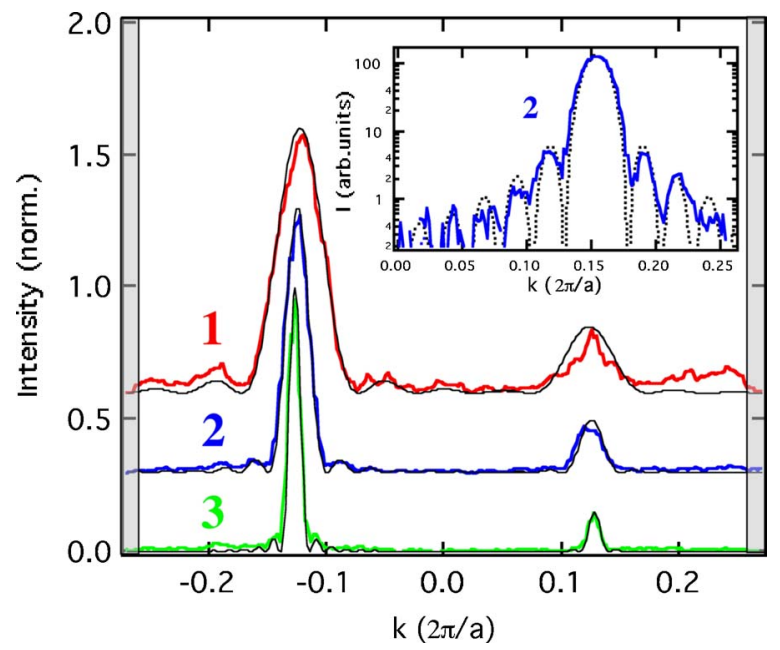

Fig. 4. (Color online) Experimental Fourier space spectra of square lattice $\mathrm{PhC}$ tiles operating in the self-collimating regime $(u=0.283)$ for TE polarization, with length: 9 (1), 18, (2), and $36 \mu \mathrm{m}$ (3). Thin dark curve: sinc fits. The spatial frequency range outside the bandwidth of the setup is highlighted in gray. Inset: log-scale plot of spectrum 2 corresponding to the $18 \mu \mathrm{m}$ long tile. 

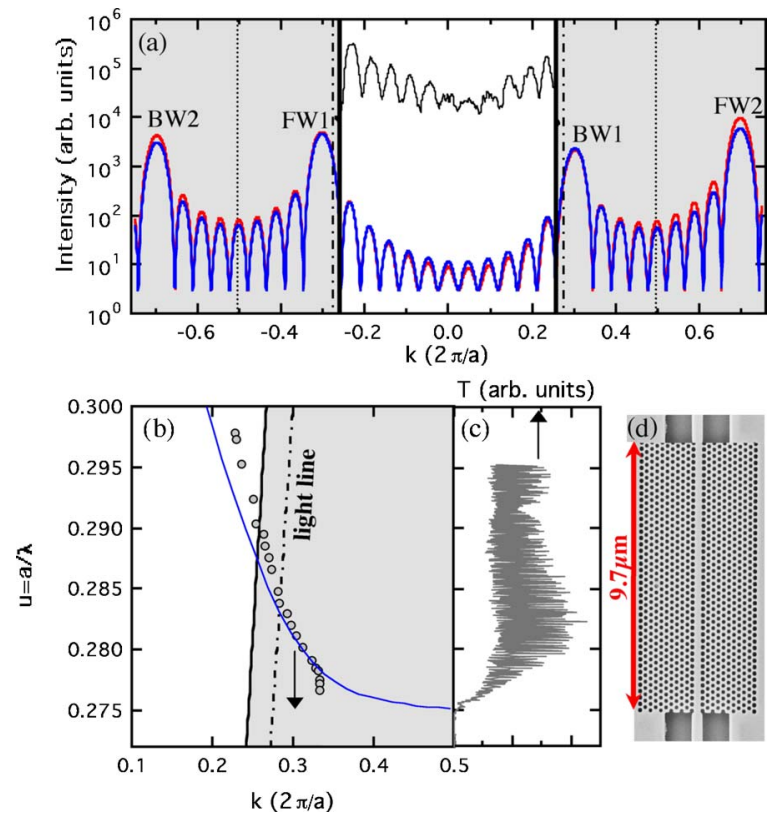

Fig. 5. (Color online) (a) Experimental Fourier space spectrum (log-scale) of a $9.7 \mu \mathrm{m}$ long W1 waveguide (dark curve), analytical continuation of the experimental spectrum (blue curve), and theoretical spectrum (red curve) at $\lambda=1565 \mathrm{~nm}(u=0.281)$. FWi: Forward propagating Bloch wave in the $i^{m e}$ Brillouin zone, BWi: Backward propagating Bloch wave in the $i^{m e}$ Brillouin zone. Dotted and dashed lines: light lines of the objective and of air, respectively. (b) Experimental dispersion curve determined from the analytical continuation of the Fourier space spectrum (circles), and theoretical dispersion curve (blue curve). (c) Endfire optical transmission through the W1 waveguide. (d) Electron microscopy image of the W1 waveguide $(a=440 \mathrm{~nm})$ structure.

maxima can be fitted by the proper sinc function to recover the position of the main peak if this one is outside the light cone. Note that the sharp decay of the field at the $\mathrm{PhC}$ interface compared to the $\mathrm{PhC}$ size guarantees the quality of the fit. In the case of a localized mode in a 2D PhC cavity, for instance the L3 cavity, the far-field spectrum is expected to differ strongly from a sinc profile, as the near-field envelope of the mode is not a square window function.

In Fig. 5 we illustrate the possibility of using the information carried by the secondary maxima with the onedimensional (1D) far-field spectrum of a single line defect (W1) $\mathrm{PhC}$ waveguide designed to operate below the light line. The structure consists of a $9.7 \mu \mathrm{m}$ long silicon-oninsulator (SOI) W1 waveguide defined in a $220 \mathrm{~nm}$ silicon layer by a line defect of 22 missing holes along the $\Gamma K$ direction in a triangular lattice array $(a=440 \mathrm{~nm})$ [see Fig. 5(d)]. As highlighted by Fig. 5(a), a structured signal (the dark curve) is measured in the far field. Such a signal comes from the partial relaxation of the spatial translation invariance of the structure. We have taken advantage of the secondary maxima to determine the dispersive properties of the $\mathrm{W} 1$ waveguide below the light line. The best fit (blue curve) of the experimental signal is obtained by taking into account the first and second components of the FW and BW reflected Bloch waves. The outputs resulting from such a procedure are the positions of the Bloch wave components in the first and second Brillouin zone, their relative weight, and the actual size of the sample. We obtain a good agreement with the theoretical spectrum (red curve), deduced from 2D plane wave expansion calculations for the positions and relative amplitudes of the Bloch wave components. The only a priori knowledge is the existence of an internal periodicity and the fact that the $\mathrm{PhC}$ is abruptly limited in size. As a result it was possible to determine the dispersion curve below the light cone as shown in Fig. 5(b) (in the same way we have extended the dispersion curve in Fig. 3 beyond the objective light line, i.e., the bandwith of the optical setup). A good agreement is achieved with the theoretical dispersion curve inferred from Fabry-Perot interferences in the $\mathrm{PhC}$ transmission calculated with the three-dimensional (3D) finite-difference time domain (FDTD). At $k=1 / 3$ $(2 \pi / a)$, the value corresponding to the maximum of the $\mathrm{PhC}$ dielectric band, the dispersion enters a regime where $k$ remains constant, probably due to the Bloch wave evanescent along the line defect and localized at the $\mathrm{PhC}$ input boundary, as also observed with local probe gratings [24]. The extrapolation of the Bloch wave component lying in the second Brillouin zone corresponds to an effective increase of the NA up to 2.5. The noise originating in the present case from a background scattering due to structural defects is the limiting factor to extend the bandwidth of the reconstructed far-field spectrum. Such an experimental technique implemented to extend the Fourier spectrum beyond the bandwidth of the instrument is related to the theoretical superresolution technique based on the analyticity of the far-field spectrum in the case of objects limited in size $[25,26]$.

\section{COUPLED CAVITY WAVEGUIDE}

As a second example of a device operating below the light line, the high NA Fourier space imaging technique is applied to a coupled cavity waveguide (CCW). CCWs are of intense current interest notably because of their potential capabilities to slow and store light pulses or to strongly reduce optical interaction lengths and enhance nonlinearity effects [27-30]. In the present case, the CCW comprises 100 linearly coupled identical cavities, each of which consists of a photonic heterostructure based on an SOI W1 PhC waveguide whose structural parameters are identical to the previously studied W1 waveguide [31]. As shown in Fig. 6(a), the supercell of the photonic heterostructure contains 11 rows of holes of lattice constant $a_{1}$ $=440 \mathrm{~nm}$ (the mirrors) and three rows of lattice constant $a_{2}=460 \mathrm{~nm}$ (the core). In Figs. 6(b) and 6(c) the propagating field is imaged in real space and Fourier space, respectively, for a wavelength of $1535 \mathrm{~nm}$ (normalized frequency $u=a_{1} / \lambda=0.2866$ ). The intensity in real space is located at the position of the cavities.

The far-field [Fig. 6(c)] consists of sharp vertical lines, labeled $d_{1}, d_{2}, d_{3}$, and $d_{4}$, and periodically spaced with a period equal to the reciprocal vector $G_{s c}=2 \pi /\left(11 a_{1}+2 a_{2}\right)$ of the CCW supercell. The spread of the radiated field in the $k_{y}$ direction is larger than in the self-collimation case and goes well beyond the objective aperture due to the extreme transverse confinement imposed by the single line $\mathrm{PhC}$ waveguide. This far-field pattern originates from the constructive interferences of 100 secondary coherent sources located at the cavity positions as observed in the 

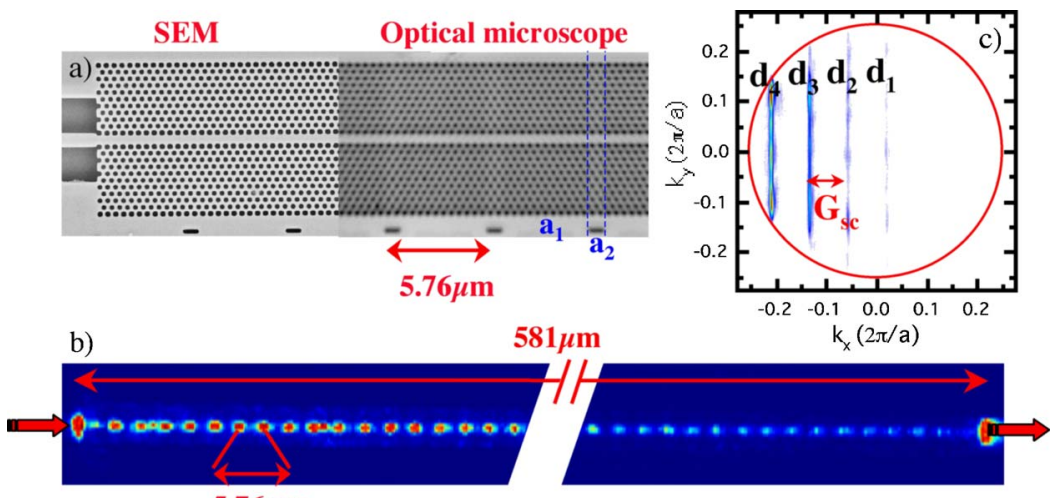

$5.76 \mu \mathrm{m}$

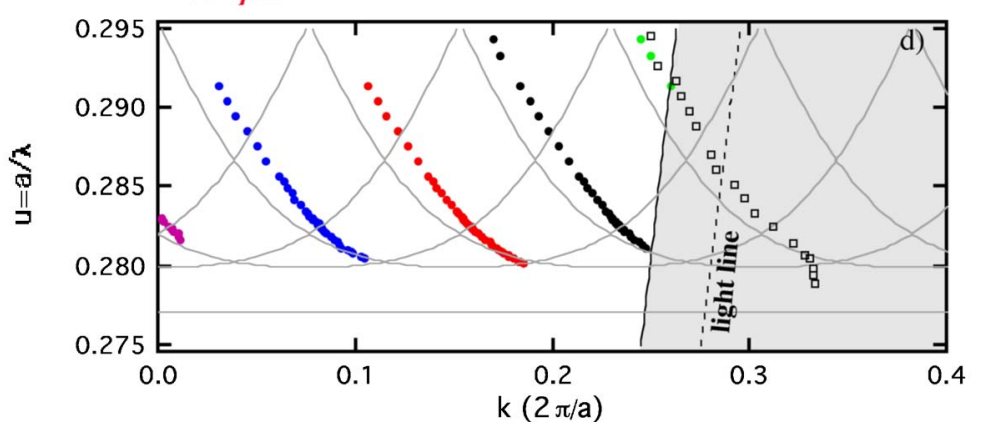

Fig. 6. (Color online) (a) Electron microscopy image of the coupled cavity waveguide, (b) and (c) real space and Fourier space images, respectively, of the CCW excited at $\lambda=1535 \mathrm{~nm}(u=0.287)$. The light radiated from the 100 cavities is observed as bright spots along the entire waveguide in real space and appears as vertical straight lines in the far field separated by the reciprocal vector $G_{s c}$ of the CCW supercell. (d) Dispersion diagram of the CCW in its first, second, third, and fourth Brillouin zones, plotted versus the modulus of the wave vector, and with $a=440 \mathrm{~nm}$ chosen for the normalization. Purple, blue, dark, and green experimental dots: position in k-space of the $d 1, d 2, d 3$, and $d 4$ lines, respectively. Square dots: experimental dispersion curve of the $9.7 \mu \mathrm{m}$ long W1 waveguide (see Fig. 3). Gray curves: theoretical 2D plane wave expansion calculations, with $30 \%$ and 2.65 as fitting parameters for the filling factor and the effective index, respectively. The deviation in the slope of the dispersion curve can be explained by the discrepancy between the idealized $2 \mathrm{D}$ model and the real 3D experiment, and not from the number of plane waves (3411) used in the model. Dotted and dashed lines: light lines of the objective and of air, respectively.

real space image. Compared with the self-collimating square lattice, where only a fraction of the first Brillouin zone is situated within the light cone, the smaller reciprocal vector $G_{s c}$ of the CCW allows us to probe four Brillouin zones inside the light cone simultaneously. The frequency dependent $k_{x}$ position of the lines $d_{1}, d_{2}, d_{3}$, and $d_{4}$ provides the dispersion curve in the first, second, third, and fourth Brillouin zones, respectively [Fig. 6(d)]. The dispersion curves have identical shapes as expected and are in good agreement with a $2 \mathrm{D}$ plane wave calculation. Note that the intensity of the component lying in the fifth Brillouin zone of the supercell is more than two decades larger than the others and that its dispersion curve (green points) is closely linked with the already measured W1 waveguide dispersion curve (open squares). As a result, with respect of the W1 guided modes, the cavities can be regarded as noninvasive spatially coherent scatterers that fold the dispersion curve of the unperturbed W1 waveguide into the light cone and allow its measurement. Due to this folding property, our measurement can therefore access parts of the $\mathrm{W} 1$ dispersion curve that are situated below the light cone. More generally, Fig. 6(d) reveals an important intrinsic property of all coupled cavities based waveguides: the periodicity of the cavities, always larger than the $\mathrm{PhC}$ pitch, implies that one or several associated Brillouin zones are entirely inside the light cone, and so some of the plane waves associated with the Bloch mode decomposition will be able to radiate. This must be considered in the design of the system to keep the contribution of these waves as low as possible and to minimize losses. A recent theoretical study by Povinelli and Fan [32] shows, for example, that the radiation loss of the coupled system may be lower than that of an individual cavity. To summarize, the measurements presented in Fig. 6 demonstrate that far-field imaging gives access to the main physical properties of $\mathrm{PhC}$ coupled cavity waveguides.

\section{LOCAL INVESTIGATION OF A PHOTONIC CRYSTAL POLARIZATION BEAM SPLITTER}

The engineering of $\mathrm{PhC}$ heterostructures offers the possibility of performing a range of optical functionalities, which will eventually be integrated as local building blocks in advanced photonic circuits on a chip. Their characterization in such a complex environment requires local probes capable of measuring their properties without disturbing the overall system. As a third example, a compact $\mathrm{PhC}$ double polarization beam splitter is presented in Fig. 7. The first splitter uses the intrinsic properties of $\mathrm{PhC}$ slabs to project the input beam polarization state on the $\mathrm{TM}$ and TE polarization states of the underlying planar waveguide. The two corresponding beams are then col- 

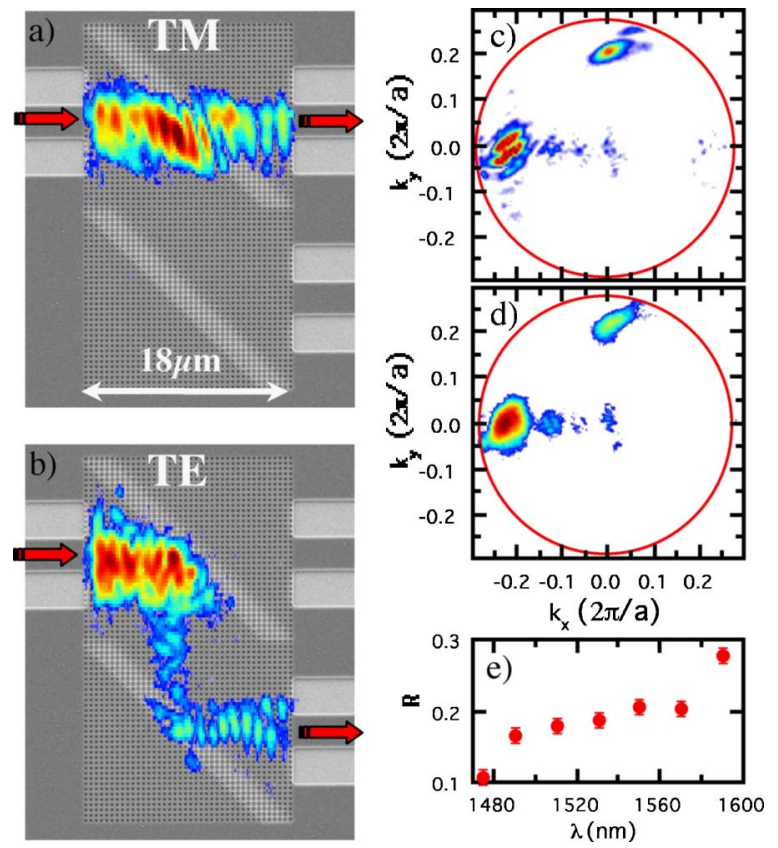

Fig. 7. (Color online) (a) and (b) near-field image of the TM and TE waves, respectively, propagating in the double slab polarizer beam splitter (color coded: increasing intensity from blue to red) superimposed with an optical microscope image of the structure (black and white), (c) emission diagram of the TE wave imaged in the Fourier space at $\lambda=1550 \mathrm{~nm}$, (d) same as (c) with the emission of the second splitter filtered in the intermediate image plane, (e) reflection coefficient of the first slab deduced from the far-field pattern measured for different wavelengths as in (c).

lected in two different output ports. The second splitter reflects the TE beams to collect the two polarizations through parallel ridge waveguides. Such an integrated polarizer is useful for implementing polarization diversity circuits, whereby the two polarizations are separated and then treated independently. Since the photonic bandgaps for $\mathrm{TE}$ and $\mathrm{TM}$ polarizations in a $\mathrm{PhC}$ span different wavelength ranges, it is possible for a given wavelength range to transmit the TM polarization (no bandgap effect) and to reflect the TE polarization (with bandgap effect). Such a splitter has been realized in a square lattice of fill factor $30 \%$ that operates in the self-collimation regime, with a central, diagonal line consisting of six holes with a fill factor of $50 \%$ acting as the polarization-sensitive power splitter. In Figs. 7(a) and 7(b) the near-field images identify the light paths associated with each polarization for a wavelength of $\lambda=1550 \mathrm{~nm}$. The TM polarized field propagates through the first splitter, as expected, whereas the TE polarized beam undergoes a reflection at each diagonal interface.

The transmission and reflection properties of each splitter cannot be extracted from the near-field images due to the overlap of incident and backscattered beams and the out-of-plane scattering signal at the different interfaces, which overlaps partly with the propagating beams at such small scales. The situation is more favorable in $k$-space. Indeed, $k$-space imaging provides a natural spatial selectivity of beams propagating along different directions, allowing the measurement of the relative intensity of each beam. For instance, in Fig. 7(c) the farfield image associated with the TE polarized beam reveals two main spots, one located at $\left(k_{x}=0, k_{y}=0.21\right)$, corresponding to the reflected beam propagating downward, and the other located at $\left(k_{x}=-0.21, k_{y}=0\right)$, corresponding to a superposition of the incident and the exiting beam that has been reflected twice. The mixing of these last two beams in $k$-space induces an interference pattern oriented along the $k_{x}=k_{y}$ axis. To separate these two contributions in the Fourier plane and estimate the reflection coefficient of the first splitter, the near field was filtered in an intermediate real space-imaging plane by blocking the radiated light that corresponds to the exiting beam. The fringe pattern then disappears in the corresponding farfield image [Fig. 7(d)], and the intensity ratio between the incident and reflected waves provides a direct quantitative measure of the beam splitter reflectivity. For the specific device shown in Fig. 7, we measure a reflectivity of $R=28 \%$ at $1.59 \mu \mathrm{m}$. This closely matches the value of $R$ $=26 \%$ obtained with a standard nonlocal end-fire transmission measurement, thus demonstrating the powerful capabilities available with our technique. Note that the secondary maxima discussed previously [see Figs. 4 and 5] are also observed in Fig. 7(d) due to the finite size of the device and as a result could be used to determine the reflection coefficient of the polarizing slab if the device would operate below the light line.

\section{CONCLUSION}

The combination of Fourier optics with a conventional end-fire setup is ideally suited to provide a clear experimental understanding of the physics of Bloch waves in periodic nanostructures. The excitation of the $\mathrm{PhC}$ structures with a narrow bandwidth laser source allows highresolution spectroscopy of the different Bloch modes, both qualitatively and quantitatively, both above and below the light line. Fundamental information such as the dispersion curves and the EFSs can rapidly be extracted from the experimental far-field images without sophisticated characterization tools and postprocessing. We envision that such experimental measurements of the EFSs on actual devices and of the light propagation or localization in nanophotonic structures opens new perspectives for implementing and developing promising concepts of nanostructures.

\section{APPENDIX: ANTENNA MODEL APPLIED TO FAR-FIELD EMISSION FROM PHC STRUCTURES}

The electric field distribution $\vec{E}_{k}(x)$ of the TM polarized Bloch mode can be expressed as $\vec{E}_{k}(\vec{r})=E_{0} u_{k}(\vec{r}) e^{j \vec{k} \vec{r}} e^{-j \omega t} \vec{z}$, with $u_{k}(\vec{r})$ a periodic function having the very periodicity of the lattice and $E_{0}$ the field amplitude. The corresponding field intensity $I \propto\left\|u_{k}(\vec{r})\right\|^{2}$ exhibits a spatial modulation equal to the periodicity of the $\mathrm{PhC}$ lattice, which is two times smaller than the present setup resolution in the infrared region. As a result, the spatially resolved modulation in the measured intensity pattern seems initially surprising. A simple model based on antenna theory can help to understand such an observation. Using the volume equivalence theorem [33], the radiated field $\vec{E}_{0}(\vec{r})$ in 
the vacuum from the $\mathrm{PhC}$ whose electrical and magnetic permittivity are respectively $\epsilon$ and $\mu_{0}$, can be calculated with the equivalent current $\vec{J}_{e q u}\left(\vec{r}^{\prime}\right)=j \omega\left(\epsilon\left(\vec{r}^{\prime}\right)-\epsilon_{0}\right) \vec{E}_{k}\left(\vec{r}^{\prime}\right)$ induced by the Bloch wave $\vec{E}_{k}\left(\vec{r}^{\prime}\right)$, with $\vec{r}^{\prime}$ the vector position in the $2 \mathrm{D}$ plane of the source and $\epsilon_{0}$ the vacuum electrical permittivity. In the first Born approximation, $\vec{E}_{k}\left(\vec{r}^{\prime}\right)$ can be chosen as the Bloch wave solution of the infinite $2 \mathrm{D} \mathrm{PhC}$. At a distance $\vec{r}$ from the sources, the expression for the radiated electric field from a TM polarized current can then be simplified as $\vec{E}_{0}(\vec{r})=\left(e^{j \overrightarrow{k_{0}} \vec{r}} / r\right) \vec{F}(\vec{\nu})$, with $\vec{F}(\vec{\nu})$ $=j \sqrt{\mu_{0} / \epsilon_{0}} \times\left(k_{0} / 4 \pi\right)[(\vec{z} \times \vec{\nu}) \times \vec{\nu}] \int_{S} J_{e q u} e^{-j \overrightarrow{k_{0}} \vec{r}^{\prime}} \mathrm{d} \vec{r}^{\prime}, \vec{k}_{0}$ the wave vector of the radiated wave, $S$ the surface defining the boundary of the equivalent courant, and $\vec{\nu}=\vec{r} / r$. This expression is simply the fundamental antenna formula [34] (for a TE polarized field the vectorial product differs, whereas the integral is unchanged). The equivalent current is also a $2 \mathrm{D}$ propagating Bloch wave with the new periodic function $\left(\epsilon\left(\overrightarrow{r^{\prime}}\right)-\epsilon_{0}\right) u_{k}\left(\vec{r}^{\prime}\right)$. By use of the periodicity of this function, $\vec{J}_{\text {equ }}\left(\vec{r}^{\prime}\right)$ can be represented as an infinite superposition of $m$ plane waves whose in-plane wave vectors are $\vec{k}_{m}=\vec{k}+\vec{G}_{m}$, with $\vec{k}$ the wave vector in the first Brillouin zone, $\vec{G}_{m}$ the reciprocal lattice vectors, and $m$ an integer. According to the expression of $\vec{F}(\vec{\nu})$, only the $m$ plane waves, for which there exists a wave vector $\vec{k}_{0}$ satisfying the conservation of the in-plane wave vector $\vec{k}_{m}$, generate a signal in the radiated field, $\vec{k}_{0}$ being limited by the light cone. The observed light can then be interpreted as a coherent emission radiated from the holes whose emission direction results from the phase coherence defined by the $2 \mathrm{D}$ propagating Bloch wave inside the $\mathrm{PhC}$. The PhC corrugation plays two major roles: (1) it acts as a distributed antenna, and (2) it distributes the energy carried by the propagating field among $m$ plane waves whose $\mathrm{k}$-components lie in the different Brillouin zones of the structure [35]. The setup aperture filters the plane waves whose $\vec{k}_{m}$ falls in the light cone of the microscope objective. In the current example we expect that an FW propagating Bloch wave would generate only one spot in the Fourier space corresponding to its only component $\vec{k}_{1}$ lying in the first Brillouin zone of the periodic lattice. The resulting image in the real space should then be a constant intensity without spatial modulation.

\section{ACKNOWLEDGMENTS}

This project was supported by the European network of excellence Epixnet IST-004525, Funfox IST-004582, the National Centre of Competence in Research-Quantum Photonics (NCCR-QP), and the European Cooperation in the Field of Scientific and Technical Research (COST) action P11. The authors thank R. Rochat for his creative technical assistance, J. D. Ganière for his constant interest and fruitful input, W. Langbein for encouraging discussion about k-space imaging, and L. A. Dunbar, H. Benisty, M. Ilegems, and B. Deveaud-Plédran for careful reading of the manuscript. The samples were fabricated in the context of an Intel Strategic Development Grant, and we thank A. Michaeli and M. Salib for their contributions.

\section{REFERENCES AND NOTE}

1. T. F. Krauss, R. M. De La Rue, and S. Brand, "Twodimensional photonic-bandgap structures operating at near-infrared wavelengths," Nature 383, 699-702 (1996).

2. X. Letartre, C. Seassal, C. Grillet, P. Rojo-Romeo, P. Viktorovitch, M. Le Vassor d'Yerville, D. Cassagne, and C. Jouanin, "Group velocity and propagation losses measurement in a single-line photonic-crystal waveguide on InP membranes," Appl. Phys. Lett. 79, 2312-2314 (2001).

3. M. Notomi, K. Yamada, A. Shinya, J. Takahashi, C. Takahashi, and I. Yokohama, "Extremely large groupvelocity dispersion of line-defect waveguides in photonic crystal slabs," Phys. Rev. Lett. 87, 253902 (2001).

4. T. Baba and T. Matsumoto, "Resolution of photonic crystal superprism," Appl. Phys. Lett. 81, 2325-2327 (2002).

5. M. J. Steel, R. Zoli, C. Grillet, R. C. McPhedran, C. Martijn de Sterke, A. Norton, P. Bassi, and B. J. Eggleton, "Analytic properties of photonic crystal superprism parameters," Phys. Rev. E 71, 056608 (2005).

6. H. Gersen, T. J. Karle, R. J. P. Engelen, W. Bogaerts, J. P. Korterik, N. F. van Hulst, T. F. Krauss, and L. Kuipers, "Direct observation of Bloch harmonics and negative phase velocity in photonic crystal waveguides," Phys. Rev. Lett. 94, 123901 (2005).

7. H. Gersen, T. J. Karle, R. J. P. Engelen, W. Bogaerts, J. P. Korterik, N. F. van Hulst, T. F. Krauss, and L. Kuipers, "Real-space observation of ultraslow light in photonic crystal waveguides," Phys. Rev. Lett. 94, 073903 (2005).

8. R. Wüest, D. Erni, P. Strasser, F. Robin, H. Jäckel, B. C. Buchler, A. F. Koenderink, V. Sandoghdar, and R. Harbers, "A standing-wave meter to measure dispersion and loss of photonic-crystal waveguides,” Appl. Phys. Lett. 87, 261110 (2005).

9. P. Kramper, M. Kafesaki, C. M. Soukoulis, A. Birner, F. Mller, U. Gsele, R. B. Wehrspohn, J. Mlynek, and V. Sandoghdar, "Near-field visualization of light confinement in a photonic crystal microresonator," Opt. Lett. 29, 174-176 (2004).

10. N. Louvion, D. Gérard, J. Mouette, F. de Fornel, C. Seassal, X. Letartre, A. Rahmani, and S. Callard, "Local observation and spectroscopy of optical modes in an active photoniccrystal microcavity," Phys. Rev. Lett. 94, 113907 (2005).

11. R. Zengerle, "Light propagation in singly and doubly periodic planar waveguides," J. Mod. Opt. 34, 1589-1617 (1987).

12. M. Galli, D. Bajoni, M. Patrini, G. Guizzetti, D. Gerace, L. C. Andreani, M. Belotti, and Y. Chen, Phys. Rev. B 72, $125322(2005)$.

13. M. Loncar, D. Nedeljkovic, T. P. Pearsall, J. Vuckovic, A. Scherer, S. Kuchinsky, and D. C. Allan, "Experimental and theoretical confirmation of Bloch-mode light propagation in planar photonic crystal waveguides," Appl. Phys. Lett. 80, 1689-1691 (2002).

14. G. Bartal, O. Cohen, H. Buljan, J. W. Fleischer, O. Manela, and M. Segev, "Brillouin zone spectroscopy of nonlinear photonic lattices," Phys. Rev. Lett. 94, 163902 (2005).

15. P. St. J. Russell, "Optics of Floquet-Bloch waves in dielectric gratings," Appl. Phys. B 39, 231-246 (1986).

16. E. Cubukcu, K. Aydin, E. Ozbay, S. Foteinopuolou, and C. M. Soukoulis, "Negative refraction by photonic crystals," Nature 423, 604-605 (2003).

17. C. Luo, S. G. Johnson, J. D. Joannopoulos, and J. B. Pendry, "All-angle negative refraction without negative effective index," Phys. Rev. B 65, 201104 (2002).

18. H. Kosaka, T. Kawashima, A. Tomita, M. Notomi, T. Tamamura, T. Sato, and S. Kawakami, "Superprism phenomena in photonic crystals," Phys. Rev. B 58, 10096-10099 (1998).

19. S. Yang, J. H. Page, Z. Liu, M. L. Cowan, C. T. Chan, and P. Sheng, "Focusing of sound in a 3D phononic crystal," Phys. Rev. Lett. 93, 024301 (2004).

20. H. Kosaka, T. Kawashima, A. Tomita, M. Notomi, T. Tamamura, T. Sato, and S. Kawakami, "Self-collimating 
phenomena in photonic crystals," Appl. Phys. Lett. 74, 1212-1214 (1999).

21. P. T. Rakich, M. S. Dahlem, S. Tandon, M. Ibanescu, M Soljacic, G. S. Petrich, J. D. Joannopoulos, L. A. Kolodziejski, and E. P. Ippen, "Achieving centimeter-scale supercollimation in a large-area two-dimensional photonic crystal," Nat. Mater. 5, 93-96 (2006).

22. R. Ferrini, A. Berrier, L. A. Dunbar, R. Houdré, M. Mulot, S. Anand, S. de Rossi, and A. Talneau, "Minimization of out-of-plane losses in planar photonic crystals by optimizing the vertical waveguide," Appl. Phys. Lett. 85, 3998-4000 (2004).

23. Note that here the $\mathrm{PhC}$ structure is excited in the second band of the dispersion diagram. As a result the group velocity in the first Brillouin zone is positive for "negative" wave vectors value, hence the image of the $\mathrm{FW}$ propagating wave in the Fourier space is located at $-k_{x}$.

24. N. Le Thomas, R. Houdré, L. H. Frandsen, J. FagePedersen, A. V. Lavrinenko, and P. I. Borel, "Gratingassisted superresolution of slow waves in Fourier space," Phys. Rev. B 76, 035103 (2007).

25. M. I. Kobolov and C. Fabre, "Quantum limits on optical resolution," Phys. Rev. Lett. 85, 3789-3792 (2000).

26. C. K. Rushforth and R. W. Harris, "Restoration, resolution, and noise," J. Opt. Soc. Am. 58, 539-545 (1968).
27. N. Stefanou and A. Modinos, "Impurity bands in photonic insulator," Phys. Rev. B 57, 12127-12133 (1998).

28. A. Yariv, Y. Xu, R. K. Lee, and A. Scherer, "Coupledresonator optical waveguide: a proposal and analysis," Opt. Lett. 24, 711-713 (1999).

29. M. F. Yanik, W. Suh, Z. Wang, and S. Fan, "Stopping light in a waveguide with an all-optical analog of electromagnetically induced transparency," Phys. Rev. Lett. 93, 233903 (2004).

30. M. F. Yanik and S. Fan, "Stopping light all-optically," Phys. Rev. Lett. 92, 083901 (2004).

31. B.-S. Song, S. Noda, T. Asano, and Y. Akahane, "Ultra-high$Q$ photonic double-heterostructure nanocavity," Astrophys. J., Suppl. Ser. 4, 93-96 (2005).

32. M. L. Povinelli and S. Fan, "Radiation loss of coupledresonator waveguides in photonic-crystal slabs," Appl. Phys. Lett. 89, 191114 (2006).

33. R. Mitra, Computer Techniques for Electromagnetics (Pergamon, 1973), Chap 2.

34. E. E. Orlova, J. N. Hovenier, T. O. Klaassen, I. Kasalynas, A. J. L. Adam, J. R. Gao, T. M. Klapwijk, B. S. Williams, S. Kumar, Q. Hu, and J. L. Reno, "Antenna model for wire lasers," Phys. Rev. Lett. 96, 173904 (2006).

35. B. Lombardet, L. A. Dunbar, R. Ferrini, and R. Houdré, "Fourier analysis of Bloch wave propagation in photonic crystals," J. Opt. Soc. Am. B 22, 1179-1190 (2005). 